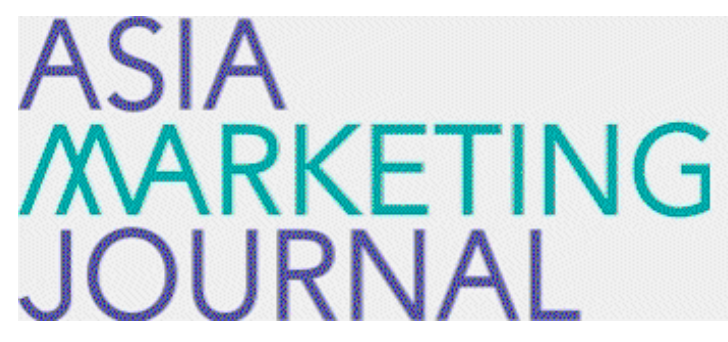

ASIA MARKETING JOURNAL

Volume 14 | Issue 4

Article 5

$1-30-2013$

\title{
A Moderating Role of Personal Need for Structure on the Effects of Process versus Outcome Simulations on the Evaluation of Really New Products
}

Jun San Kim

Min Yeosun Yoon

Min Hi Hahn

Follow this and additional works at: https://amj.kma.re.kr/journal

Part of the Marketing Commons

\section{Recommended Citation}

Kim, Jun San; Yoon, Min Yeosun; and Hahn, Min Hi (2013) "A Moderating Role of Personal Need for Structure on the Effects of Process versus Outcome Simulations on the Evaluation of Really New Products," Asia Marketing Journal: Vol. 14 : Iss. 4 , Article 5.

Available at: https://doi.org/10.53728/2765-6500.1501

This Article is brought to you for free and open access by Asia Marketing Journal. It has been accepted for inclusion in Asia Marketing Journal by an authorized editor of Asia Marketing Journal. 


\title{
A Moderating Role of Personal Need for Structure on the Effects of Process versus Outcome Simulations on the Evaluation of Really New Products*
}

\author{
Jun San Kim** \\ Minhi Hahn*** \\ Yeosun Yoon ${ }^{* * * *}$
}

Really new products (RNPs) provide novel benefits yet many consumers are reluctant to accept these highly innovative new products. Previous literature has shown that mental simulation is an effective method for enhancing the evaluation of RNPs. However. Castano et al. (2008) and Zhao, Hoeffler, and Zauberman (2011) demonstrate conflicting results as to which type of mental simulation (i.e., process versus outcome) is more effective for the enhancement of RNP evaluation. The authors try to reconcile these results by incorporating a moderating variable which is personal need for structure (PNS). PNS is an individual difference variable that taps the differences in people's propensity to cognitively structure and simplify their environment (Neuberg and Newsom 1993). From the analysis of the previous two works, the authors point out that consumers susceptibility to uncertainty may contribute to the different results, and suggest that this susceptibility is dependent on consumers PNS. To test the hypotheses established, an experiment was conducted. Waterless washing machine was presented as a RNP and PNS was measured by using the 12-item PNS Scale (Thompson et al. 2001). The results of the study show that for high-PNS consumers, process simulation is more effective than outcome simulation for enhancing the evaluation of a RNP. whereas for low-PNS consumers, outcome simulation is more effective than process simulation.

This research contributes to the mental simulation and new product literature by suggesting and verifying that PNS moderates the effects of process versus outcome simulations for enhancing the evaluation of RNPs. This research provides important managerial implications for marketing managers of RNPs, indicating that they should take account of the target consumers' PNS in planning marketing

\footnotetext{
* This article is based in part on the doctoral dissertation of Jun San Kim.

The authors thank dissertation committee members Yong Jin Hyun. Youngbae Kim. and Jae-Hyeon Ahn. and Jongbae Kim. Changjo Yoo. and three anonymous AMJ reviewers for their constructive comments and suggestions.

** Senior Researcher. College of Business, KAIST(strider@business,kaist,ac,kr), corresponding author

*** Professor of Marketing. College of Business, KAIST(mhhahn@business.kaist.ac.kr)

**** Associate Professor of Marketing. College of Business. KAIST(yyoon@business.kaist.ac.kr)
} 
communications, Specifically, when targeting high-PNS consumers, marketing communications that encourage process simulation may be more effective than those that encourage outcome simulation. In contrast, when targeting low-PNS consumers, marketing communications that encourage outcome simulation may be more effective than those that encourage process simulation.

Key words: really new products, process simulation, outcome simulation, personal need for structure, susceptibility to uncertainty

Innovative new products such as iPad, Slate $\mathrm{PC}$, and robotic products are considered as newly developed products that have not been existed, that is, really new products (RNPs) (Hoeffler 2003). Firms often devote considerable efforts to develop these innovative new products to ensure their profitability and sustainable growth. However, it is a well known fact that the success rate of RNPs is exceedingly low (Gourville 2006), calling for more research regarding the ways to enhance the evaluation of these highly novel products (e.g.. Wind and Mahajan 1997). As one of the methods, mental simulation has been found effective (e.g., Dahl and Hoeffler 2004: Hoeffler 2003: Zhao, Hoeffler, and Dahl 2009). However, previous literature shows conflicting results as to which type of mental simulation (i.e., process versus outcome) is more effective than the other. That is, the results of the studies by Castano et al. (2008) show that process simulation is more effective than outcome simulation, whereas the results of the studies by Zhao, Hoeffler, and Zauberman (2011) show that outcome simulation is more effective than process simulation. This research tries to extricate these contrary results by suggesting a moderator which is personal need for structure (PNS).

PNS is an individual difference variable that taps the differences in people's propensity to cognitively structure and simplify their environment (Neuberg and Newsom 1993). Recently, PNS has been identified as a moderator that affects the relationship between the degree of product newness and consumers' new product evaluation (Kim, Hahn, and Yoon 2012). Likewise, this research suggests that PNS plays a moderating role on the effects of process versus outcome simulations for enhancing consumers' evaluation of RNPs. To this end, we analyze the previous two works and suggest that the more effective type of mental simulation differs according to consumers' susceptibility to uncertainty. Also, based on the previous literature (Landau et al. 2006: Landau et al. 2004: Neuberg and Newsom 1993: van den Bos et al. 2005), we propose that consumers' 
susceptibility to uncertainty is positively related to consumers' PNS. Based on these assertions, we predict that for high-PNS consumers, process simulation is more effective than outcome simulation, whereas for low-PNS consumers, outcome simulation is more effective than process simulation. We verify these predictions by an experiment which show supportive results for the hypotheses. These results provide an explanation for the conflicting results of the previous literature. Also, they indicate that marketing managers of RNPs should take account of the target consumers' PNS in deciding the type of mental simulation, which may be embedded in marketing communications for RNPs.

This article is organized as follows. First, we briefly review the literature about enhancing the evaluation of RNPs. Among them, the effects of mental simulation are detailed and conflicts surrounding them are investigated. Second, a moderating role of PNS is suggested regarding which type of mental simulation is more effective for enhancing the evaluation of RNPs. Third, an empirical study was conducted to test the hypotheses established and the results of the study are provided. This article concludes with discussions about the results of the study and managerial implications for marketing managers.

\section{Mental Simulation and the Evaluation of Really New Products}

\subsection{Enhancing the Evaluation of Really New Products}

Really new products (RNPs) are the new products with novel benefits creating a new product category (Gregan-Paxton and John 1997: Hoeffler 2003), such as iPad. They enable consumers to do things that could not be done with the existing products. However, consumers are more uncertain about the performance of RNPs than the existing products and they often require substantial learning and change of behavior for consumers to effectively use them. And these characteristics are believed to lower the acceptance of RNPs (Alexander, Lynch, and Wang 2008: Castano et al. 2008: Hoeffler 2003: Hoeffler and Herzenstein 2011: Zhao, Hoeffler, and Dahl 2009). This gave rise to the stream of literature that explored the ways to enhance the evaluation of RNPs (e.g., Dahl and Hoeffler 2004: Herzenstein, Posavac, and Brakus 2007: Jhang, Grant, and Campbell 2011: Zhao, Hoeffler, and Dahl 2009). Researchers have suggested several methods for this goal. For example, the inducement of promotion focus has been shown to enhance the adoption of RNPs compared to the inducement of pre- 
vention focus (Herzenstein, Posavac, and Brakus 2007). Also, the facilitation of cognitive flexibility has been shown to enhance the evaluation of extremely incongruent new products (Jhang, Grant, and Campbell 2012). As one of the ways, the effects of mental simulation has received wide attention among researchers (e.g., Castano et al. 2008: Hoeffler 2003: Zhao, Hoeffler, and Zauberman 2007: Zhao, Hoeffler, and Zauberman 2011). However, a debate remains about which type of mental simulation (i.e., process versus outcome) is more effective for enhancing the evaluation of RNPs than the other. After we introduce mental simulation and its types, we investigate more details regarding this issue.

\subsection{Effects of Process versus Outcome Simulations on the Evaluation of Really New Products}

Mental simulation is the imitative representation of some event or series of events (Taylor and Schneider 1989). It represents a mental construction of real or hypothetical events. Researchers have classified mental simulation into two types: process versus outcome (Taylor et al. 1998). Process simulation is a simulation with an emphasis on the step-by-step process needed to reach a goal. Outcome simulation is a simulation with emphasis on the resulting outcome a person would gain when the goal is achieved. The differential effects of these two types of mental simulation have been widely studied in the domain of social psychology (e.g., Pham and Taylor 1999: Rivkin and Taylor 1999: Taylor and Pham 1999). For example, process simulation has been shown to be more effective than outcome simulation in facilitating studying and improving grades (Pham and Taylor 1999). In marketing, researchers have proposed that process simulation refers to a simulation of the step-by-step process of using the product, while outcome simulation refers to a simulation of the end benefits one would gain and enjoy after using the product (Escalas and Luce 2003, 2004). Also, they have shown that process simulation elicits higher purchase intention than outcome simulation. As above examples indicate, researchers have showed that process simulation is more effective than outcome simulation for the achievement of some goals or for the inducement of positive consumer responses toward products.

However, in terms of the enhancement of consumers' RNP evaluation, there have been conflicting results as to which type of mental simulation is more effective. That is, Castano et al. (2008) have shown that process simulation is more effective than outcome simulation for increasing adoption intention for RNPs, whereas Zhao, Hoeffler, and Zauberman (2011) have reported that outcome simulation is more effective than process simulation for increasing the evaluation of RNPs. Although they have 
incorporated a number of factors other than the type of mental simulation, we contend that their results differ in the conditions that are similar to each other.

As for the research by Castano et al. (2008), they assert that the more effective type of mental simulation differs by temporal distance (near versus distant) to the adoption of RNPs. The focus of this article is on consumers on-line evaluation as they're exposed to RNPs. Thus, we only consider the results in the near future condition from the studies by Castano et al. (2008). In this condition, they show that process simulation is more effective than outcome simulation.

As for the research by Zhao, Hoeffler, and Zauberman (2011), they did not use the term RNPs in their research. However, product stimuli presented to participants in their studies were mostly RNPs (e.g., Sony tablet, Apple iPad). Moreover, one of the product stimuli, AudioPC. was the same product as the one presented as a RNP in the previous research (cf., Zhao, Hoeffler, and Dahl 2009). Thus, we contend that the results of their studies show the effects of mental simulation on the evaluation of RNPs. They assert that the more effective type of mental simulation differs by the type of information processing mode of mental simulation (cognitive versus affective), product type (utilitarian versus hedonic) and temporal distance (near versus distant). As we stated earlier, we only consider the results in the near future condition. However, for the information processing mode and product type, we consider the results in the specific conditions that match the studies by Castano et al. (2008).

Zhao, Hoeffler, and Zauberman (2011) argue that previous literature on mental simulation has combined both cognitive and affective processing modes of mental simulation. They distinguish between these two processing modes by the specific emphasis given by the mental simulation instructions, that is, whether the instruction asks participants to focus on their thoughts or feelings. The mental simulation instructions used in the studies by Castano et al. (2008) emphasize participants' thoughts, rather than feelings. Thus, we contend that the results of the studies by Castano et al. (2008) show the effects of cognitive processing mode of mental simulation. Moreover, new product stimuli presented to participants in the studies by Castano et al. (2008) were utilitarian products (e.g., virtual courseware and automatic car). Thus, we only consider the results in the cognitive processing mode, the utilitarian product and the near future conditions from the studies by Zhao Hoeffler, and Zauberman (2011). In these conditions, they show that outcome simulation is more effective than process simulation.

To summarize these arguments, the results of the previous two works differ in the similar conditions as to which type of mental simulation is more effective for enhancing the evaluation of RNPs. We contend that Castano et 
al. (2008) give emphasis on the cognitive dimension of mental simulation and focus on the utilitarian products. Given this, Castano et al. (2008) show that process simulation is more effective than outcome simulation in the near future condition. However, Zhao, Hoeffler, and Zauberman (2011) show that outcome simulation is more effective than process simulation in the cognitive processing mode, the utilitarian product, and the near future conditions. This research tries to explicate this conflict, and thus, we only consider the results in the aforementioned conditions from the studies of the previous two works.

Regarding the contrary results, Zhao, Hoeffler, and Zauberman (2011) point out that the mental simulation instructions are different between the two works. In a different perspective, we suggest that the more effective type of mental simulation for enhancing the evaluation of RNPs differs depending on consumers susceptibility to uncertainty. And we propose that this susceptibility to uncertainty is dependent on consumers' individual difference of PNS. Before we arrive at these assertions, we investigate the differences between the assertions of the previous two works.

\section{A Moderating Role of Personal Need for Structure on the Effects of Mental Simulation}

\subsection{Differences between the Two Works}

In this section, we analyze the differences in the assertions by Castano et al. (2008) and Zhao, Hoeffler, and Zauberman (2011). Note that the analysis is restricted to the conditions set in the previous section. Castano et al. (2008) suggest that uncertainty is the major barrier for consumers' adoption of RNPs. Among various types of uncertainty, they assert that switchingcost uncertainty plays the most important role. Switching-cost uncertainty refers to the uncertainty consumers perceive about how much time and effort would be needed for learning how to use the new products (Castano et al. 2008). They suggest that simulating the stepby-step process of using the product will alleviate this switching-cost uncertainty, increasing the adoption intention for RNPs. Thus, they predict and show that process simulation is more effective than outcome simulation for enhancing consumers' adoption of RNPs.

Similarly, Zhao, Hoeffler, and Zauberman (2011) suggest that consumers focus on the learning costs of RNPs. However, they do not mention uncertainty in their studies. They assert that consumers' focus on costs, rather than benefits, is the major barrier for the 
adoption of RNPs. They suggest that simulating the outcome, or the end benefits after using the product will change consumers' focus from costs to benefits, enhancing the evaluation of RNPs. Thus, they predict and show that outcome simulation is more effective than process simulation for enhancing the evaluation of RNPs.

The above analysis comes to a finding of this research regarding the differences between the previous two works. They both posit that consumers focus more on the process (i.e., learning costs) than outcome (i.e., benefits of using the product) of RNPs when they're exposed to them. However, they differ in their assertions regarding the effect of uncertainty on consumers adoption of RNPs. Castano et al. (2008) assert that it is affected by uncertainty regarding the learning costs related to RNPs (i.e., switching-cost uncertainty). In contrast, Zhao, Hoeffler, and Zauberman (2011) argue that it is not affected by uncertainty, even though consumers focus on the learning costs related to RNPs.

As Hoeffler (2003) stated, RNPs are characterized as having more uncertainty than the existing products. Thus, the characteristics of consumers, which is related to their proneness to the effect of uncertainty (i.e., consumers' susceptibility to uncertainty), may settle the different assertions of the previous two works. That is, consumers' susceptibility to uncertainty may lead to the different adoption processes of RNPs. Specifically, for consumers who are more susceptible to uncertainty, process simu- lation may be more effective than outcome simulation by alleviating switching-cost uncertainty. However, for consumers who are less susceptible to uncertainty, outcome simulation may be more effective than process simulation by switching their focus from costs to benefits. Thus, we suggest that the more effective type of mental simulation for enhancing the evaluation of RNPs differs according to consumers' susceptibility to uncertainty. And we propose that this consumers' susceptibility to uncertainty is dependent on consumers' personal need for structure.

\subsection{Personal Need for Structure and the Effects of Mental Simulation}

Personal need for structure (PNS) is an individual difference construct that taps the extent to which people are inclined to simplify and structure their environment (Neuberg and Newsom 1993: Thompson et al. 2001). People simplify and structure their world to make it more manageable, because they cannot process all the information they receive (Neuberg and Newsom 1993). And some of them have a stronger need for this simple structuring than others. PNS has been widely researched in the domain of social psychology and revealed to affect people's various interpersonal behaviors such as stereotyping and trait inference (e.g., Moskowitz 1993: Neuberg and Newsom 1993: Schaller et al. 1995). 
Previous literature has not revealed the relationship between consumers' susceptibility to uncertainty and PNS. However, we infer this from the results of the previous literature (Landau et al. 2006: Landau et al. 2004: Neuberg and Newsom 1993: van den Bos et al. 2005). Researchers have shown that PNS moderates the effect of mortality salience: high-PNS individuals are more affected by mortality salience than low-PNS individuals (Landau et al. 2006: Landau et al. 2004). Specifically, under mortality salience, high-PNS individuals seek structures by relying on the representative information, whereas low-PNS individuals do not show these tendencies (Landau et al. 2004). Also, when mortality thoughts are salient, preference for the seemingly meaningless modern arts declines among high-PNS individuals, whereas no such effect is evident among low-PNS individuals (Landau et al. 2006). These results can be interpreted alternatively according to the assertions by van den Bos et al. (2005). They contend that mortality salience and uncertainty salience prompt similar reactions, suggesting that uncertainty salience may be an important part of mortality salience manipulation. Thus, the results of the studies by Landau et al. (2004) and Landau et al. (2006) can be interpreted as showing the moderating role of PNS on the effect of uncertainty: high-PNS individuals are more susceptible to uncertainty. whereas low-PNS individuals are less susceptible to uncertainty. Moreover, PNS has been shown to be positively related to intolerance of ambiguity (Budner 1962: Neuberg and Newsom 1993). That is, high-PNS individuals are more threatened by ambiguous situations than lowPNS individuals. From the above arguments, we suggest that consumers' susceptibility to uncertainty is positively related to consumers' PNS.

We summarize our arguments to arrive at two hypotheses. We suggest that the more effective type of mental simulation for enhancing the evaluation of RNPs differs according to consumers' susceptibility to uncertainty. Specifically, we suggest that for consumers who are more susceptible to uncertainty, process simulation is more effective than outcome simulation, whereas for consumers who are less susceptible to uncertainty, outcome simulation is more effective than process simulation. Also, we propose that consumers' susceptibility to uncertainty is positively related to consumers PNS. Thus, we predict that for high- PNS consumers, process simulation is more effective for enhancing the evaluation of RNPs than outcome simulation, whereas for low-PNS consumers, outcome simulation is more effective than process simulation. These predictions can be formally described as follows:

H1: High-PNS consumers give a higher eval-
uation to a really new product after proc-
ess simulation than outcome simulation.
H2: Low-PNS consumers give a higher eval- 
uation to a really new product after outcome simulation than process simulation.

\section{Study}

An experiment with the two conditions (mental simulation: process versus outcome) was designed to test the predictions established in the previous section. Participants were randomly assigned to one of the two conditions.

\subsection{Method}

\subsubsection{Participants and Procedure}

Ninety-one female undergraduate and graduate students from the universities in the Republic of Korea participated in the experiment. They were given a booklet which contained an advertisement of a RNP and measures. The advertisement was followed by an instruction for mental simulation focused either on the process or the outcome of using the advertised product. After responding the measures, participants were debriefed and compensated.

\subsubsection{Stimulus}

An advertisement for a RNP was developed as the stimulus. A new product that may create a new subordinate level category within the basic level category was provided as the RNP (Kim, Hahn, and Yoon 2012). The experimenter made the decision as to whether the new product will create a new subordinate category or not. A washing machine category was selected as the basic level category. As the new product, a waterless washing machine which uses polymer beads to wash garments instead of water and detergent was presented. This new product was under development and was not commercially available at the time of the experiment. The waterless washing machine was expected to create a new subordinate level category within the washing machine category because of its unique feature. Because the attitude toward a washing machine category could differ across gender, only the female students were recruited to participate in the study. The advertisement presented a picture of a washing machine, the headline, and the product description. The new product was presented with a fictitious brand name Nespia. The headline of the advertisement read. "Revolutions of Washing Machine! Nespia ${ }^{\circledR}$ Waterless Washing Machine."

\subsubsection{Simulation Instruction}

The mental simulation instructions were constructed after the instructions used in the previous literature (Escalas and Luce 2003: Zhao, Hoeffler, and Dahl 2009: Zhao, Hoeffler, and Zauberman 2007). Participants in the process simulation condition read the following instruction. 
The reason why you use a washing machine is to wash garments.

Imagine that you wash garments using Nespia ${ }^{(0)}$ Waterless Washing Machine while thinking about the process and the procedure you need to go through from the moment you purchase Nespia Waterless Washing Machine to the moment you finish washing the garments.

That is, think about the process you would go through to arrive at the goal of "washing the garments" using Nespia Waterless Washing Machine.

Participants in the outcome simulation condition read the following instruction.

The reason why you use a washing machine is to wash garments.

Imagine that you wash garments using Nespia ${ }^{\text {(i) }}$ Waterless Washing Machine while thinking about the outcome and the benefits you earn and enjoy when you wash garments using Nespia ${ }^{(1)}$ Waterless Washing Machine.

That is, think about the outcome you would earn and enjoy when you arrive at the goal of "washing the garments" using Nespia ${ }^{\circledR}$ Waterless Washing Machine.

To guide participants' mental simulation, the examples of thoughts generated from the simulation are provided (e.g., Castano et al. 2008). To stimulate mental simulation toward the advertised new product, the examples of the different product category (i.e., wireless mouse) were provided. To facilitate mental simulation, we asked participants to list their thoughts generated from the simulation.

\subsubsection{Measures}

As a dependent variable, product evaluation was measured by using the four nine-point items anchored by "very bad / very good," "very poor / very excellent," "very negative / very positive," and "very unfavorable / very favorable," which were averaged to form the overall evaluation ( $a=.93$ ). As a manipulation check, product newness was measured by using the four ninepoint items anchored by "not at all new / very new," "not at all novel / very novel," "not at all innovative / very innovative," and "not at all original / very original" (Herzenstein, Posavac, and Brakus 2007: Zhao, Hoeffler, and Dahl 2009), which were averaged to form the overall product newness score $(a=.97)$. As a manipulation check measure for process simulation, participants were asked to rate how much they thought about the process and the procedure of using the advertised product on a nine-point item anchored by "not at all thought about the process and the procedure / very much thought about the process and the procedure." As a manipulation check measure for outcome simulation, participants were asked to rate how much they thought about the outcome and the benefits of using the advertised product on a nine-point item anchored by "not at all thought about the outcome and the benefits / very much thought about the outcome and the benefits." As an independent variable, PNS was measured by using the 12-item PNS Scale (Thompson et 
al. 2001). The items were averaged to form participants' PNS score $(a=.82)$. Also, participants' performance uncertainty ratings toward the advertised new product was measured to verify the relationship between PNS and consumers' susceptibility to uncertainty. Although our argument focuses on the switchingcost uncertainty. we measured performance uncertainty because this type of uncertainty is known to be the most prevalent one among various types of uncertainty in RNPs (Hoeffler 2003), and our aim here is to provide initial verification for the relationship between PNS and consumers' susceptibility to uncertainty. Participants were asked to rate how certain about how much the advertised product will be useful to them on a nine-point item anchored by "very uncertain about how much useful this will be / very certain about how much useful this will be" (Castano et al. 2008). As a demographic variable, participants' age was measured.

\subsection{Results}

Participants rated the advertised new product newer than the midpoint $(\mathrm{M}=7.04$. $\mathrm{t}(90)=10.41: p<.001)$. Thus, product newness was manipulated as intended. Participants in the process simulation condition responded that they focused on the process during mental simulation more than participants in the outcome simulation condition $\left(\mathrm{M}_{\text {Process }}=5.76\right.$,
$\left.\mathrm{M}_{\text {Outcome }}=5.02: \mathrm{t}(89)=1.97: p=.051\right)$. Participants in the outcome simulation condition responded that they focused on the outcome during mental simulation more than participants in the process simulation condition $\left(\mathrm{M}_{\text {Outome }}=6.47, \mathrm{M}_{\text {Process }}=5.43: \mathrm{t}(89)=\right.$ 2.51: $p=.014)$. Thus, mental simulation was manipulated as intended.

To test $\mathrm{H} 1$ and $\mathrm{H} 2$, regression was used (Irwin and McClleland 2001). Product evaluation was regressed by mental simulation dummy variable (process simulation coded as 0 ), PNS, and their interaction, and revealed significant interaction effect $(p=.041)$. For high-PNS participants, product evaluation was regressed by mental simulation, PNS (adjusted to the high-level of PNS by 2 SD: Irwin and McClleland 2001), and their interaction. The sign of beta coefficient of mental simulation was minus as predicted $(b=-490)$ and the effect was significant $(p=.049)$. For low-PNS participants, product evaluation was regressed by mental simulation, PNS (adjusted to the lowlevel of PNS by 2 SD: Irwin and McClleland 2001), and their interaction. The sign of beta coefficient of mental simulation was plus as predicted $(b=390)$ and the effect was significant $(p=.091)$. Thus, both $\mathrm{H} 1$ and $\mathrm{H} 2$ were supported by the results of the study. Figure 1 shows the cell means using a median split for PNS (median $=3.92$ ). To test the relationship between PNS and consumers' susceptibility to uncertainty, participants' perform- 
ance uncertainty ratings were regressed by PNS. The sign of beta coefficient of PNS was plus as predicted $(b=.221)$ and the effect was significant $(p=.003)$. Thus, the results of the study showed that PNS is positively related to consumers' susceptibility to uncertainty.

The study was conducted to test the hypotheses established about a moderating role of PNS on the effects of process versus outcome simulations for enhancing the evaluation of RNPs. Specifically, we predicted that for high-PNS consumers, process simulation is more effective than outcome simulation, whereas for low-PNS consumers, outcome simulation is more effective than process simulation. The results of the study supported both predictions. Also, the results of the study provided initial evidence regarding the positive relationship between PNS and susceptibility to uncertainty.

\section{Discussion}

In this research, we tried to explicate the contrary results within the existing literature regarding the effects of mental simulation on the evaluation of RNPs by incorporating a moderating variable, which is personal need for structure (PNS). Previously, Castano et al. (2008) have found that process simulation is more effective than outcome simulation, whereas Zhao, Hoeffler, and Zauberman (2011) have shown that outcome simulation is more effective than process simulation. We propose that the more effective type of mental simulation

〈Figure 1) Study Results:

Evaluation of a RNP After Process Versus Outcome Simulations as a Function of PNS

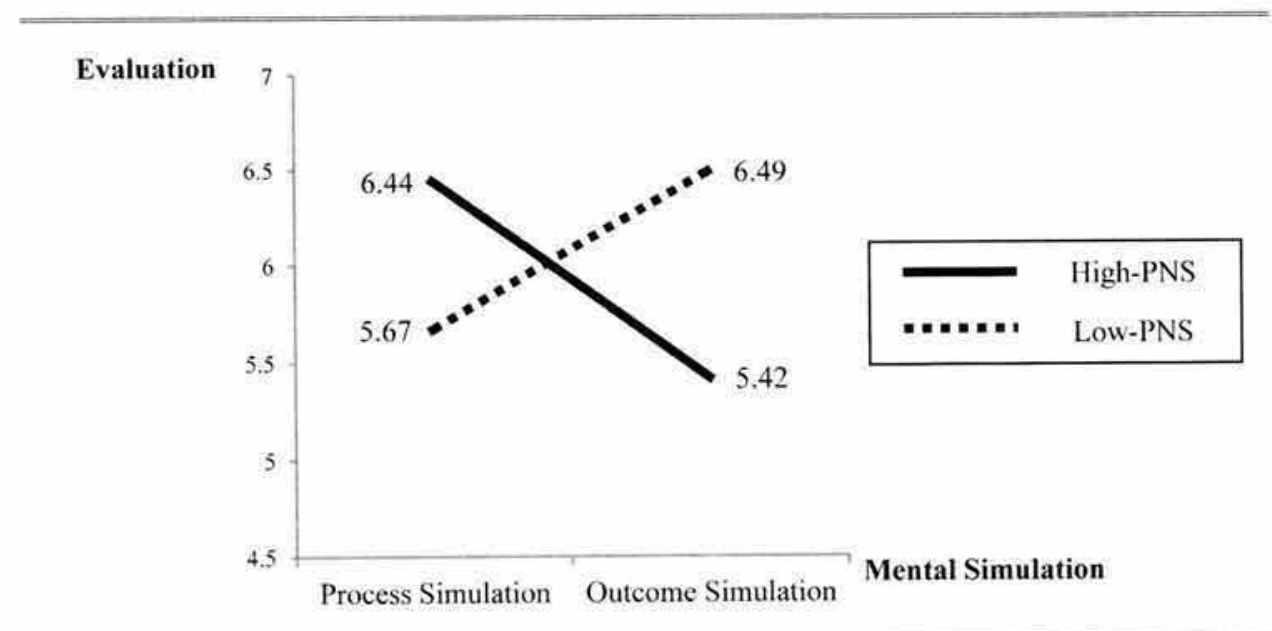

Note: Scores reported are condition means using median split for PNS. 
differs according to consumers' susceptibility to uncertainty. And we suggest that consumers' susceptibility to uncertainty is dependent on consumers' PNS, which is an individual difference variable regarding people's propensity to simplify and structure their environment (Neuberg and Newsom 1993). The results of the study show that for high-PNS consumers, process simulation is more effective than outcome simulation, which are consistent with the results of studies by Castano et al. (2008). In contrast, for low-PNS consumers, they show that outcome simulation is more effective than process simulation, which are consistent with the results of studies by Zhao, Hoeffler, and Zauberman (2011). Thus, this research contributes to the mental simulation and new product literature by suggesting and verifying that PNS moderates the effects of process versus outcome simulations for enhancing the evaluation of RNPs.

The results of this research provide an alternative explanation for the conflicting results of the previous two works by Castano et al. (2008) and Zhao, Hoeffler, and Zauberman (2011). Zhao, Hoeffler and Zauberman (2011) suggest that the differences in the mental simulation instructions between the two works may be one of the reasons for the conflicting results. They argue that the mental simulation instructions in their studies are directionoriented, whereas those used in the studies by Castano et al. (2008) are solution-oriented (i.e.. including examples and guides). In this research, the mental simulation instructions were constructed based on the ones used by both works. That is, participants were provided with the instructions accompanied by the specific examples that guided their simulation. Although the instructions were the same across participants' PNS, the results of the study show that the more effective type of mental simulation differs according to their PNS. This indicates that the conflicting results between the previous two works may be in part due to the differences in participants' PNS. Although participants were randomly assigned to one of the experimental conditions, those in the studies by Castano et al. (2008) were recruited from the universities in Mexico, whereas those in the studies by Zhao, Hoeffler, and Zauberman (2011) were recruited from the universities in the US. This could systematically affect participants' PNS, resulting in the different results.

As a future research, the underlying mechanism regarding the moderating role of PNS on the effects of process versus outcome simulations awaits further investigation. We assert that consumers may go through the different adoption processes of RNPs according to their susceptibility to uncertainty, or PNS. That is, for high-PNS consumers, process simulation may be more effective than outcome simulation by alleviating switching-cost uncertainty, whereas for low-PNS consumers, outcome simulation may be more effective than process simulation 
by switching their focus from costs to benefits. Investigation of these processes will enlighten the underlying mechanism as well as consumers' new product adoption processes and the role of PNS on them (e.g.. Moreau, Lehmann, and Markman 2001). As another future research. the relationship between PNS with other variables including demographic and psychographic factors needs further investigation. As mentioned above, regional, cultural, and national characteristics may affect consumers' PNS. Also, marketing managers may find it difficult to identify low- or high-PNS consumers. Thus. exploring the relationship between consumers' PNS and variables which marketing managers often use to segment consumers is another promising future research question.

As a limitation of the research, we focused on the results in the specific conditions from the previous two works. That is, we only considered the results in the near future condition from the studies by Castano et al. (2008) and the results in the cognitive processing mode, the utilitarian product, and the near future conditions from the studies by Zhao, Hoeffler, and Zauberman (2011). Investigating the effects of PNS in relation with these factors will help uncover the larger picture regarding the effect of mental simulation on the evaluation of RNPs. For example, the role of PNS on the effects of mental simulation in the affective processing mode or for the hedonic products may be investigated in the future. As another limitation, the explicit use of mental simulation instructions hinders the results of the study to be generalized in the real marketing communications. Thus, it would be a meaningful extension of this research to provide participants with the ad-embedded type of mental simulation (e.g., Zhao. Hoeffler, and Zauberman 2011). This will be particularly helpful for marketing managers. Finally, the study employed only one product category, a washing machine. Replicating the results of the study for the products in the other product categories will increase the external validity of the study results.

This research provide important managerial implications for marketing managers of RNPs. Because failure rates of RNPs are notoriously high (Gourville 2006: Schneider and Hall 2011), firms can benefit by adopting various methods to enhance consumers' evaluation of RNPs. Previous literature suggests several methods to fulfill this goal. For example, Jhang, Grant, and Campbell (2012) suggest that positive emotional appeals may enhance the evaluation of RNPs. As for mental simulation, researchers have suggested that emphasizing the process or the outcome of using the product in marketing communications may enhance the evaluation of RNPs (e.g., Castano et al. 2008: Dahl and Hoeffler 2004: Zhao, Hoeffler, and Dahl 2009: Zhao, Hoeffler, and Zauberman 2011). However, it has not been clear that which type of mental simulation is more effective. The results of the study suggest that marketing managers should 
choose the type of mental simulation in their marketing communications based on the target consumers' PNS. That is, when targeting highPNS consumers, marketing communications that encourage process simulation may be more effective than those that encourage outcome simulation. In contrast, when targeting low-PNS consumers, marketing communications that encourage outcome simulation may be more effective than those that encourage process simulation. Previous literature suggest that marketing managers of new products may benefit by taking account of consumers' goals (Park, Kim, and Chung 2012). Likewise, the results of this research indicate that they should consider the target consumers' PNS in planning their marketing communications.

This research investigated the role of individual difference of PNS on the effects of process versus outcome simulations for enhancing the evaluation of RNPs. The results of the study highlight that considering consumers' individual characteristics is important for both academics and marketing managers of RNPs. As Ziamou (1999) has suggested, investigating the effect of consumers' individual differences on their responses to RNPs will increase the understanding regarding the nature of consumers' adoption processes as well as the success factors of RNPs.

〈Received September 20. 2012〉

〈Revised November 27. 2012〉

〈Accepted December 6. 2012〉

\section{References}

Alexander, David L., John G. Lynch, and Qing Wang (2008). "As Time Goes By: Do Cold Feet Follow Warm Intentions for Really New Versus Incrementally New Products?," Journal of Marketing Research, 45 (3). 307-19.

Budner, Stanley (1962), "Intolerance of Ambiguity as a Personality Variable," Journal of Personality, 30 (1), 29-50.

Castano, Raquel, Mita Sujan, Manish Kacker, and Harish Sujan (2008), "Managing Consumer Uncertainty in the Adoption of New Products: Temporal Distance and Mental Simulation," Journal of Marketing Research, 45 (3), 320-36.

Dahl, Darren W. and Steve Hoeffler (2004), "Visualizing the Self: Exploring the Potential Benefits and Drawbacks for New Product Evaluation," Journal of Product Innovation Management, 21 (4), 259-67.

Escalas, Jennifer Edson and Mary Frances Luce (2003), "Process Versus Outcome Thought Focus and Advertising," Journal of Consumer Psychology, 13 (3), 246-54. and (2004), "Understanding the Effects of Process-Focused versus OutcomeFocused Thought in Response to Advertising," Journal of Consumer Research, 31 (2), 274-85 Gourville, John T. (2006), "Eager Sellers \& Stony Buyers," Harvard Business Review, 
84 (6), 98-106.

Gregan-Paxton, Jennifer and Deborah Roedder John (1997), "Consumer Learning by Analogy: A Model of Internal Knowledge Transfer," Journal of Consumer Research, 24 (3), 266-84.

Herzenstein, Michal, Steven S. Posavac, and J. Joško Brakus (2007), “Adoption of New and Really New Products: The Effects of Self-Regulation Systems and Risk Salience," Journal of Marketing Research, 44 (2), 251-60. Hoeffler, Steve (2003), "Measuring Preferences for Really New Products," Journal of Marketing Research, 40 (4), 406-20.

and Michal Herzenstein (2011). "Optimal Marketing for Really New Products: Using a Consumer Perspective to Improve Communications," in Cracking the Code: Leveraging Consumer Psychology to Drive Profitability, Steven S. Posavac, ed. NY: M. E. Sharpe, Inc.

Irwin, Julie R. and Gary H. McClelland (2001), "Misleading Heuristics and Moderated Multiple Regression Models," Journal of Marketing Research. 38 (1), 100-09.

Jhang, Ji Hoon, Susan Jung Grant, and Margaret C. Campbell (2012), “Get It? Got It. Good! Enhancing New Product Acceptance by Facilitating Resolution of Extreme Incongruity," Journal of Marketing Research, 49 (2). $247-59$.

Kim, Jun San, Minhi Hahn, and Yeosun Yoon (2012), "A Moderating Role of Personal
Need for Structure on the Evaluation of Incrementally New Products Versus Really New Products," Unpublished Manuscript, College of Business, Korea Advanced Institute of Science and Technology.

Landau, Mark J., Jeff Greenberg, Sheldon Solomon, Tom Pyszczynski, and Andy Martens (2006), "Windows into Nothingness: Terror Management, Meaninglessness, and Negative Reactions to Modern Art," Journal of Personality and Social Psychology, 90 (6), 879-92. , Michael Johns, Jeff Greenberg, Tom Pyszczynski, Andy Martens, Jamie L. Goldenberg, and Sheldon Solomon (2004), "A Function of Form: Terror Management and Structuring the Social World," Journal of Personality and Social Psychology, 87 (2), 190-210.

Moreau, C. Page, Donald R. Lehmann, and Arthur B. Markman (2001), "Entrenched Knowledge Structures and Consumer Response to New Products," Journal of Marketing Research, 38 (1), 14-29.

Moskowitz, Gordon B. (1993), "Individual Differences in Social Categorization: The Influence of Personal Need for Structure on Spontaneous Trait Inferences," Journal of Personality and Social Psychology, 65 (1), 132-42.

Neuberg, Steven L. and Jason T. Newsom (1993). "Personal Need for Structure: Individual Differences in the Desire for Simple Structure," Journal of Personality and Social 
Psychology, 65 (1), 113-31.

Park, Sehoon, Moon-Yong Kim, and Minhyung Chung (2012), "Effects of Interpretation Strategies and Consumers' Goals on Consumers Response to Hybrid Products," Asia Marketing Journal, 13 (4), 1-27.

Pham, Lien B. and Shelley E. Taylor (1999), "From Thought to Action: Effects of ProcessVersus Outcome-Based Mental Simulations on Performance," Personality and Social Psychology Bulletin, 25 (2), 250-60.

Rivkin. Inna D. and Shelley E. Taylor (1999), "The Effects of Mental Simulation on Coping with Controllable Stressful Events," Personality and Social Psychology Bulletin, 25 (12), 1451-62.

Schaller, Mark, Carrie Boyd, Jonathan Yohannes, and Meredith O'Brien (1995), "The Prejudiced Personality Revisited: Personal Need for Structure and Formation of Erroneous Group Stereotypes," Journal of Personality and Social Psychology, 68 (3), 544-55.

Schneider, Joan and Julie Hall (2011). "Why Most Product Launches Fail," Harvard Business Review, 89 (4), 21-3.

Taylor, Shelley E.. Lien B. Pham, Inna D. Rivkin, and David A. Armor (1998), "Harnessing the Imagination: Mental Simulation, SelfRegulation, and Coping," American Psychologist. 53 (4), 429-39. and Lien B. Pham (1999) "The Effect of Mental Simulation on Goal-Directed Performance," Imagery and Human Develop- ment. 18 (4), 253-68. and Sherry K. Schneider (1989), "Coping and the Simulation of Events," Social Cognition, 7 (2), 174-94.

Thompson, Megan. M.. Michael. E. Naccarato, Kevin. C. H. Parker, and Gordon B. Moskowitz (2001), “The Personal Need for Structure (PNS) and Personal Fear of Invalidity (PFI) Scales: Historical Perspectives, Present Applications and Future Directions," in Cognitive Social Psychology: The Princeton Symposium on the Legacy and Future of Social Cognition, G. Moskowitz. ed. NJ: Lawrence Erlbaum Associates, 19-39. van den Bos, Kees, P. Marijn Poortvliet, Marjolein Maas, Joost Miedema, and Ernst-Jan van den Ham (2005), "An Enquiry Concerning the Principles of Cultural Norms and Values: The Impact of Uncertainty and Mortality Salience on Reactions to Violations and Bolstering of Cultural Worldviews," Journal of Experimental Social Psychology, 41 (2), 91-113.

Wind, Jerry and Vijay Mahajan (1997), "Editorial: Issues and Opportunities in New Product Development: An Introduction to the Special Issue," Journal of Marketing Research, 34 (1), 1-12.

Zhao, Min, Steve Hoeffler, and Darren W. Dahl (2009), "The Role of Imagination-Focused Visualization on New Product Evaluation," Journal of Marketing Research, 46 (1), 46-55. 
, and Gal Zauberman (2007),

"Mental Simulation and Preference Consistency over Time: The Role of ProcessVersus Outcome-Focused Thoughts," Journal of Marketing Research, 44 (3), 379-88. and (2011), "Mental Simulation and Product Evaluation: The
Affective and Cognitive Dimensions of Process Versus Outcome Simulation," Journal of Marketing Research, 48 (5), 827-39. Ziamou, Paschalina (1999), "The Effect of the Degree of Newness of a 'Really New' Product on Consumers' Judgments," Advances in Consumer Research, 26 (1), 368-71. 